\title{
HIGH CHARGE EFFECTS IN SILICON DRIFT DETECTORS WITH LATERAL CONFINEMENT OF ELECTRONS*
}

\author{
Andrea Castoldi ${ }^{1}$ and Pavel Rehak ${ }^{2}$ \\ ${ }^{1}$ Universita degli Studi Di Milano, Milano, Italy \\ ${ }^{2}$ Brookhaven National Laboratory, Upton, NY 11973
}

October, 1995

${ }^{*}$ Work supported in part by the U.S. Department of Energy Contract No. DE-AC02-98CH10886. 


\title{
High Charge Effects in Silicon Drift Detectors with Lateral Confinement of Electrons
}

\author{
Andrea Castoldi ${ }^{1}$ and Pavel Rehak ${ }^{2}$ \\ 'Università degli Studi di Milano, Dipartimento di Fisica, via Celoria 16, Milano 20133 Italy \\ ${ }^{2}$ Brookhaven National Laboratory, Upton. 11973 New York
}

\section{Abstract}

A new drift detector prototype which provides suppression of the lateral diffusion of electrons has been tested as a function of the signal charge up to high charge levels, when electrostatic repulsion is not neglegible. The lateral diffusion of the electron cloud has been measured for injected charges up to $2 \cdot 10^{5}$ electrons. The maximum number of electrons for which the suppression of the lateral spread is effective is obtained.

\section{INTRODUCTION}

A new drift detector design has been recently proposed [1] which prevents the free diffusion of the electron cloud in the direction transverse to the drift. The control of the lateral width of the electrons is achieved by an array of $p^{+}$deep implants parallel to the drift direction that act as rigid guidelines during the drift of the charge cloud. The electron cloud, generated by an ionising event, can broaden freely only in the initial part of the motion, while it approaches the potential minimum placed close to the implanted surface. During the following part of the motion the charge distribution is "frozen" into the drift channels and is carried without further brodening and irrespective of the drift distance to the read-out anodes. This implies that the potential barrier between adjacent drift channels generated by the ionized impurities of the deep implants is high enough along the whole drift path to assure electron confinement. This requires a careful design of the distance from the implanted surface at which the electron trajctory is placed. Mutual repulsion between the electrons in fact sets a threashold value of the charge above which the confining action of the lateral potential barrier is (partially) overcome.

In order to optimize the performance of this kind of detectors the maximum charge that can be succesfully confined within a drift channel for a given potential barrier must be investigated. We here present the obtained experimental results.

\section{DRIFT DETECTOR GEOMETRY}

Differently from the "classical" drift detector [2], here a much lower negative potential is applied to the anode side of the detector than on the opposite side of the wafer. The trajectory of the charge cloud is thus displaced close to the implanted surface (anode side), where the small potential barrier generated by the ionised impurities of the deep implants is able to confine the electrons within two neighbour channel-stop's during the drift. The biasing of the device is facilitated by drifting the electrons within an epitaxial layer with higher doping than the bulk so that the depth of the electron trajectory is mainly dependent on the voltages of the anode side. Fig.I shows the metalization mask of the active area and guard area of the drift detector prototype (anode side) used in the experiment.

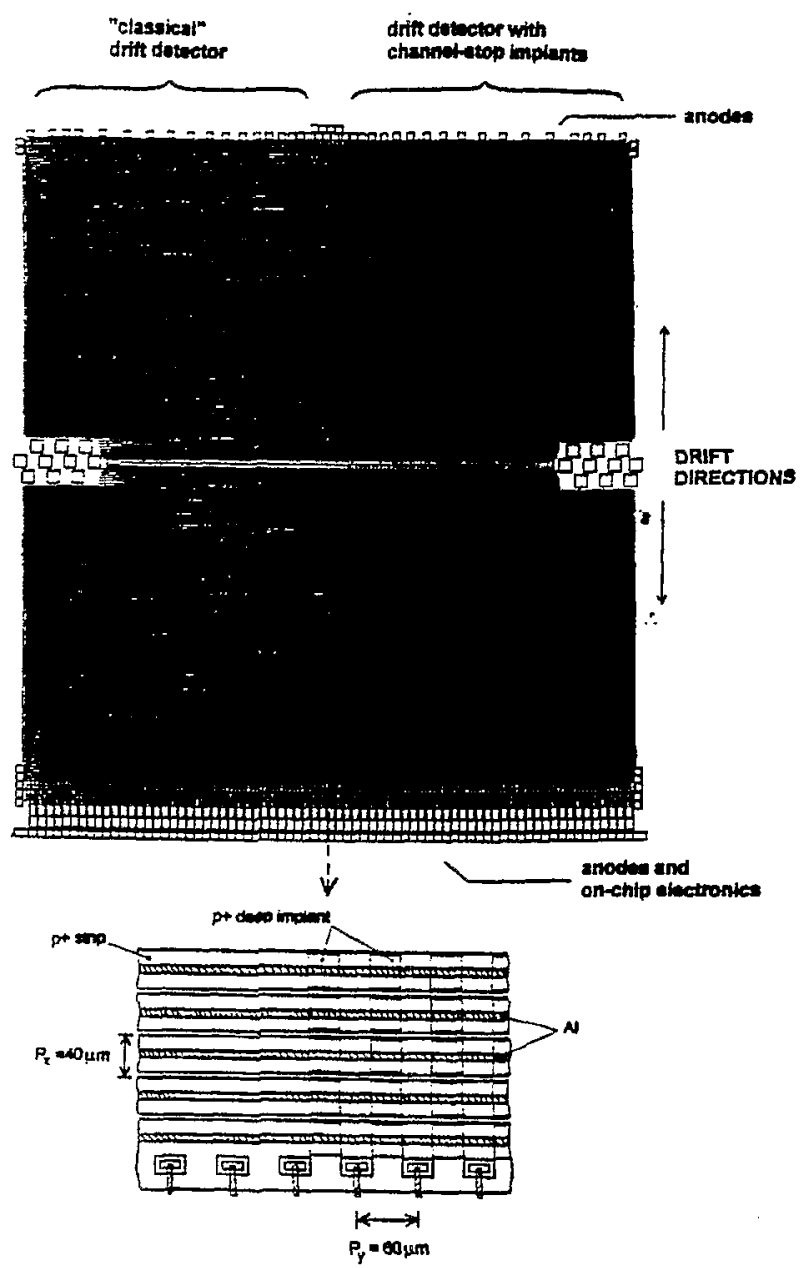

Fig.1:Metalization mask of the drift detector (anode side). Details of the channel-stop deep implants near the collecting anodes are also reported 
An array of p+ deep implants ("channel-stop's") parallel to the drift direction with pitch $P_{y}=60 \mu \mathrm{m}$ is realised in the right half of the active area while in the left half the cloud can broaden freely like in a classical drift detector. In order to avoid a possible reduction of threshold for the onset of the punch-through current between the shallow p+ strips, the regions of deep implant are interrupted in correspondence of the oxide gaps between the $p+$ strips. The drift detector project was carried through as a parasitic test on the production of the fully depleted CCD for the XMM satellite mission [3]. The prototype of the drift detector conformed to the starting silicon epitaxial material and to all production steps of the CCD production.

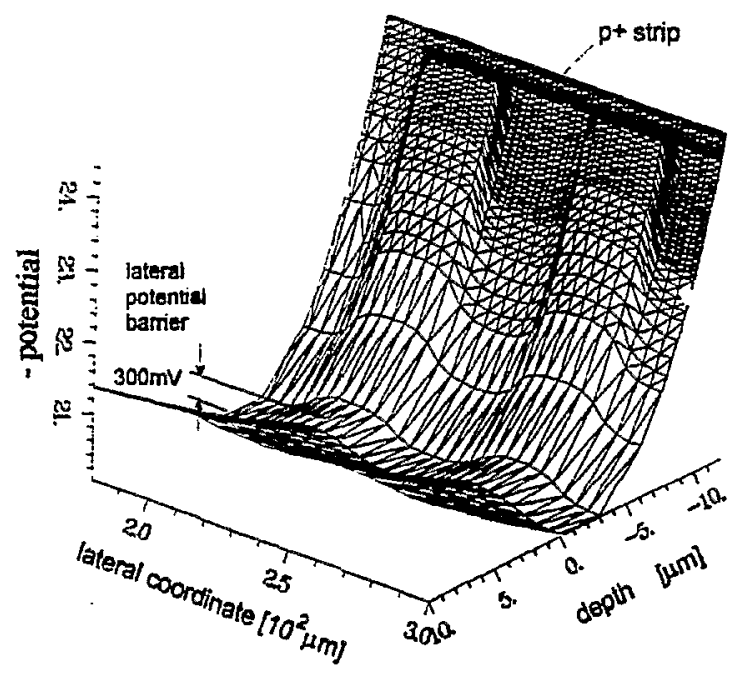

Fig.2 Potential energy for the electrons (negative potential) in a detector cross-section perpendicular to the drift. The effects of two regions of deep implant is visible.

In order to investigate how the implanted ionised impurities control the potential near the surface, we used a two-dimensional device simulator which solves the complete system of semiconductor equations [4]. Fig.2 shows the calculated potential energy of the electrons (negative potential) in a detector cross-section perpendicular to the drift direction. The channel-stop implant (Boron, $480 \mathrm{keV}$, dose $=2 \cdot 10^{11} \mathrm{~cm}^{-3}$ ) is in electric contact with the $\mathrm{p}+$ shallow implant due to a high density layer of holes and. apart from a built-in drop, it takes the potential of the $p^{+}$strip on the surface. In the minimum of the potential energy, placed at the interface between substrate and epitaxy $(z=0)$, a lateral potential barrier $\Delta V_{b} \cong 300 \mathrm{mV}$ for electron confinement is obtained.

\section{EXPERIMENT}

A pulsed semiconductor laser $(904 \mathrm{~nm})$ with pulse duration of the order of few nanoseconds was used to generate the signal electrons. The laser intensity was adjusted to generate from $10^{3}$ up to $2 \cdot 10^{5}$ electrons. The laser spot was focused by microscope optics on the anode side of the detector. In this way electrons are generated directly into the drift channel so that the initial part of the motion towards the potential minimum and the consequent spread are practically canceled. The design of the detector prototype allows to investigate, in the same detector sample, the diffusive properties of the electron cloud in presence or absence of a lateral confining field.
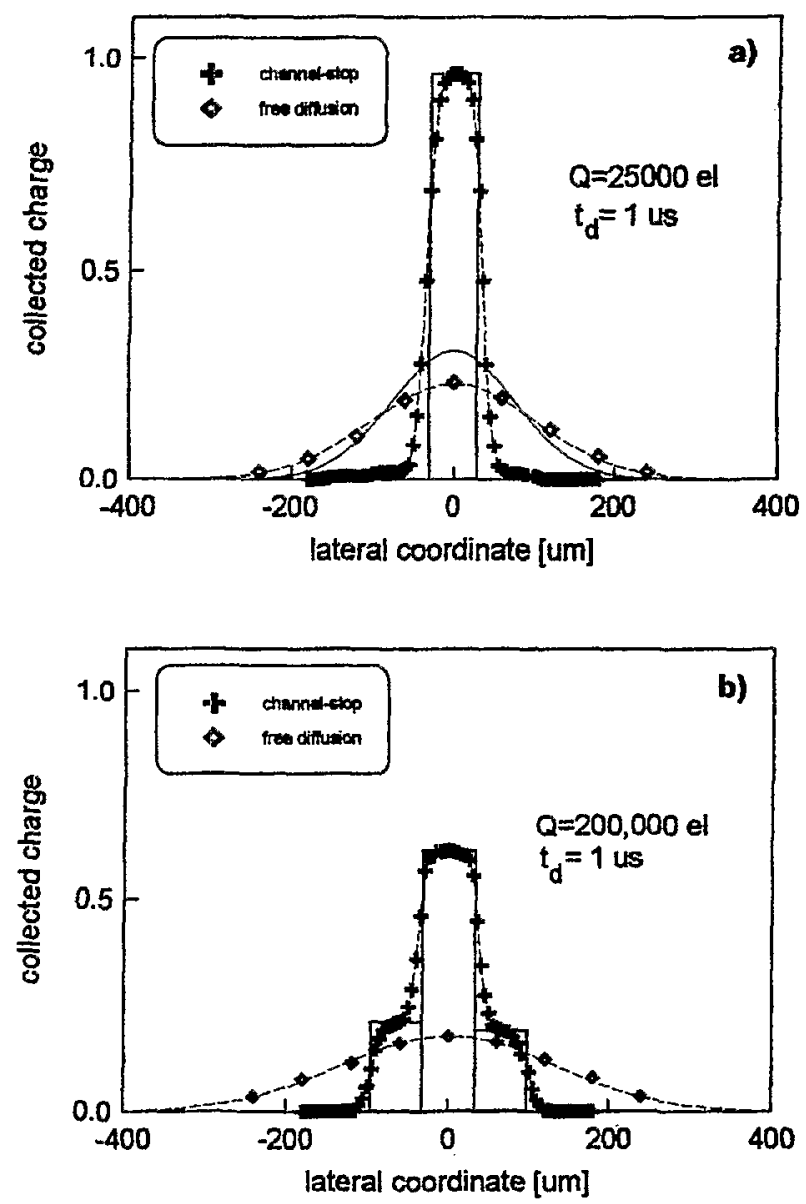

Fig.3: Collected charge at one anode as a function of the lateral position of injection after $1 \mu s$ drift time. The two cases when the anode is in the channel-stop region and when it is in the region of free broadening are compared (a) $Q=25,000$ electrons. The solid line is the solution of the continuity equation for pure diffusion; (b) $Q=200,000$ electrons. 
Measurements of the lateral broadening of the electron cloud are obtained by sampling the collected charge along the lateral coordinate. A sample interval smaller than the anode pitch is achieved by measuring the charge collected at the same anode for different lateral displacements of the laser spot.

In Fig. 3a,3b the electron broadening in presence and absence of the channel-stop implants is reported after lus drift time. At low charge levels ( $Q \leq 25,000$ electrons) all the injected charge is confined within the starting drift channel. The smooth transitions near the edges of the drift channel are due to the finite size of the laser spot. De-convolution of the experimental clata from the rectangular weighting function of the anode gives an estimate of the spot size $(\sim 15 \mu \mathrm{m})$ in agreement with independent optical measurements. The corresponding free broadening case spreads over several anodes and already shows a small extra-broadening due to repulsion. The effect of electrostatic repulsion becomes more evident at $Q=200,000$ electrons (see Fig. 3b). Here the threashold charge for succesfil confinement is clearly trespassed and the electrons start to fill the first-neighbour channels. It must be noted that the second-neighbour channel still remain empty so that a significant reduction of the lateral width of the electron cloud with respect to the free broadening case is achieved anyway. A set of similar measurements has been done for different drift times (from $0.4 \mu \mathrm{s}$ to $1 \mu \mathrm{s}$ ) by varying the drift distance. The lateral profile of the collected charge results practically unchanged, confirming that the redistribution of the electrons takes place in the first instants after generation when the mutual repulsion is dominant, then the charge distribution is "frozen" in the drift channels.

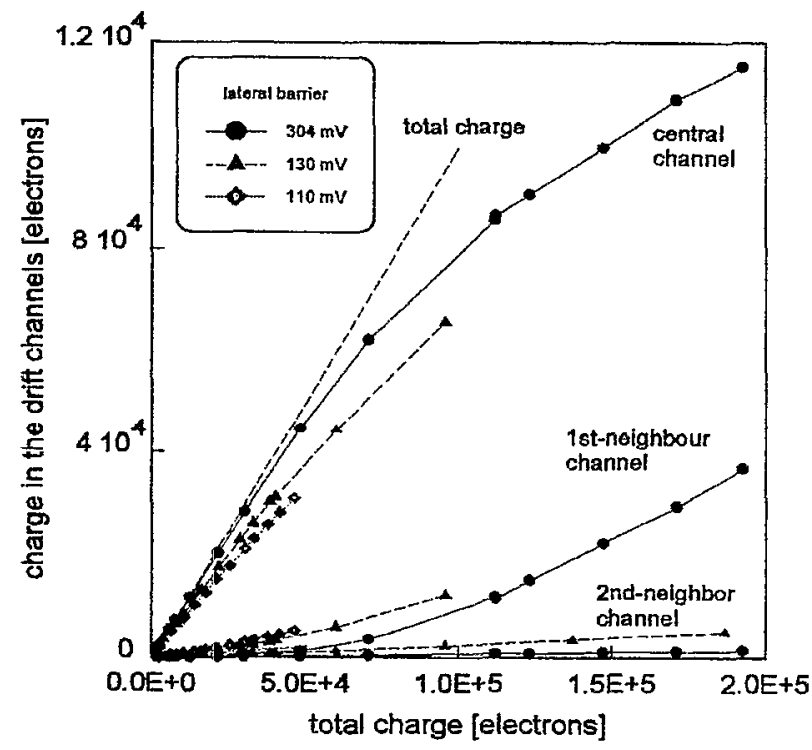

Fig.4: Measurement of the charge collected at the central and at the nearest channels as a function of the total charge.
Fig. 4 shows how the total injected charge is shared between the central and the nearest channels when the total charge is varied finely up to $2 \cdot 10^{5}$ electrons. The results for three different bias conditions, corresponding to different distance of the electron trajectory from the surface, are reported for comparison. The corresponding potential barriers are obtained from the simulated potential distributions. In all experimented cases for small number of electrons the charge collected at the nearest channels is neglegible and the charge of the central channel follows the dashed line. At higher charge levels the departure of the central charge from the ideal behaviour and the consequent increase of the firstneighbour channels is evident. The threashold values can be obtained from the data when the charge of the central channel drops below $99 \%$ of the total charge. In the case $\Delta V_{b}=304 \mathrm{mV}$ we get $Q_{\text {th }} \sim 32,000$ electrons, while $Q_{\text {th }}$ drops to 4,300 and 2,720 electrons in the cases $\Delta V_{b}=130 \mathrm{mV}$ and $110 \mathrm{mV}$ respectively. As pointed out before, the second-neighbour channel still collect a neglegible fraction, and will start to be filled only at a higher threashold.

A first interpretation of the experimental threshold charges can be obtained by the following electrostatic model. We assign to each electron, in addition to its thermal energy $V_{t h}$, a term accounting for the average repulsion energy. The average electrostatic energy per electron $q V_{\text {rep }}$, for $N$ electrons uniformly distributed inside a sphere of radius $R$, is expressed by:

$$
V_{\text {rep }}=\frac{q}{4 \pi \varepsilon} \frac{3}{5} \frac{N}{R}
$$

which shows the relationship between the total number of electrons, the size of the confinement and the potential barrier. Assuming that $\left(V_{t h}+V_{\text {rep }}\right)=1 / 3 \Delta V_{b}$ for successful confinement and solving Eq.1 for $N$, an approximate threshold of $3 \cdot 10^{4}$ electrons is obtained for $\Delta V_{b}=300 \mathrm{mV}$ and $R=P y / 2=30 \mu \mathrm{m}$. Such estimate is already in good agreement with the experimental values.

\section{CONCLUSIONS}

The effectiveness of the channel-stop implants to confine the signal electrons in parallel drift channels has been tested as a function of the signal charge, and for different positions of the electron trajectory corresponding to different lateral barriers.

The experimental results show that the electron cloud remains fully confined within a $60 \mu \mathrm{m}$ wide drift channel with $300 \mathrm{mV}$ lateral barrier throughout the drift at charge levels up to 32,000 electrons. With a lateral barrier of $110 \mathrm{mV}$ the threashold charge drops down to 2,720 electrons. An electrostatic model is reported which is in good agreement with the experimental values and shows the interrelationships between the pitch of the drift channels, the threashold charge 
above which repulsion overcomes the confining field and the lateral potential barrier.

We acknowledge M.Morelli and C.Guazzoni for help during the experimental measurements.

This manuscript has been authored under contract number DE-AC02-76CH00016 with the U.S. Department of Energy. Accordingly, the U.S. Government retains a non-exclusive, royalty free licence to publish or reproduce the published form of this contribution, or allow others to do so, for U.S. Government purposes. This work was also supported by the Italian INFN, MURST and CNR.

\section{REFERENCES}

[1] A.Castoldi and P.Rehak, 7th European Symposium on Semiconductor Detectors, Schloss Elmau, Germany, May 7-10, 1995.

[2] P.Rehak, et al., Nucl. Instr: and Meth., A248 (1986) 367378.

[3] H.Brauninger, et al., Nucl. Instr, and Meth., A326 (1993) 129-135.

[4] G.Baccarani, R.Guerrieri, P.Ciampolini and M.Rudan, "HFIELDS: a highly flexible 2-D semiconductor-device analysis program", Nasecode IV, Dublin, Ireland, June 19-21, 1985 . 